CARTA AL EDITOR

\section{RECOMENDACIONES PARA DESCRIBIR DE FORMA ADECUADA UNA CURVA EPIDÉMICA DE COVID-19}

\section{RECOMMENDATIONS TO ADEQUATELY DESCRIBE AN EPIDEMIC CURVE FOR COVID-19}

\section{Steev Loyola (iD) ${ }^{1, a}$, Andree Valle ${ }^{2}{ }^{2, b}$, Stephanie Montero ${ }^{30, c}$, Gabriel Carrasco-Escobar (iD) 4,5,d}

1 Facultad de Medicina, Universidad Peruana Cayetano Heredia, Lima, Perú.

2 Facultad de Ciencias Biológicas, Universidad Nacional Mayor de San Marcos, Lima, Perú.

3 Unidad de Investigación en Enfermedades Emergentes y Cambio Climático, Universidad Peruana Cayetano Heredia, Lima, Perú.

4 Instituto de Medicina Tropical Alexander von Humboldt, Universidad Peruana Cayetano Heredia, Lima, Perú.

5 División de enfermedades infecciosas, Universidad de California, San Diego, La Jolla, California, Estados Unidos.

a Tecnólogo médico, magíster en Epidemiología; b bachiller en Genética y Biotecnología; ' bióloga microbióloga parasitóloga; ${ }^{\mathrm{d}}$ biólogo, magíster en Epidemiología.

Sr. Editor: A finales de diciembre de 2019 apareció en China un nuevo conoravirus, que causa la enfermedad de coronavirus (COVID-19) en humanos ${ }^{(1)}$. El 6 de marzo de 2020 se presentó el primer caso confirmado de COVID-19 en el Perú. Desde entonces, el Ministerio de Salud (MINSA) comunica diariamente cuál es la situación epidemiológica.

En múltiples redes sociales hemos observado un interés continuo por construir diariamente curvas epidemiológicas para estimar la «dinámica» de COVID-19, usando incidencia acumulada, tasa de incidencia y «predicciones» basadas en tendencias. En ese sentido, nuestro objetivo es describir conceptos clave (Material suplementario), supuestos y limitaciones que deben considerarse para describir y analizar adecuadamente una curva epidémica. Ejemplificaremos estos puntos usando los datos obtenidos en China (excepto Hubei) y Japón ${ }^{(2)}$. La metodología está disponible en GitHub ${ }^{(3)}$.

El número de casos confirmados reportados diariamente depende de factores, como i) la definición de caso sospechoso según el MINSA; ii) las políticas y estrategias sanitarias; iii) la capacidad para realizar más pruebas diagnósticas; iv) el desempeño diagnóstico de las pruebas; y v) las vulnerabilida-

Citar como: Loyola S, Valle A, Montero S, Carrasco-Escobar G. Recomendaciones para describir de forma adecuada una curva epidémica de COVID-19. Rev Peru Med Exp Salud Publica. 2020;37(2):378-80. doi: https://doi.org/10.17843/ rpmesp.2020.372.5461

Correspondencia: Steev Loyola; Av. Honorio Delgado 430, Urb. Ingeniería, San Martín de Porres, Lima, Perú; steev.loyola@gmail.com

Recibido: 02/04/2020 Aprobado: 13/04/2020 En línea: 22/04/2020 des preanalíticas en laboratorio. En tal sentido, la tendencia observada en una gráfica de casos positivos no debería interpretarse estrictamente como incidencia acumulada, sino como porcentaje de casos confirmados del total de sospechosos analizados.

Del mismo modo, observamos con preocupación el desarrollo de «modelos predictivos» usando regresiones que analizan casos acumulados. En este contexto, los modelos mecanísticos son útiles en el campo de la epidemiología por su naturaleza dinámica y su poder predictivo ${ }^{(4)}$. Recientemente, en Japón se evaluó y pronosticó la propagación de COVID-19 en diversos escenarios mediante un modelo mecanístico que consideró parámetros relacionados con el ciclo natural de la infección, tasas de transición entre compartimentos, composición poblacional, entre otros ${ }^{(5)}$. En tal sentido, sugerimos que los «modelos predictivos» basados en regresiones sean interpretados como una proyección ajustada a datos observados con problemas de autocorrelación temporal, y no como información de la dinámica de la epidemia ${ }^{(4,6)}$.

Hemos observado que se usa con frecuencia la curva de casos incidentes para calcular la tasa de incidencia de COVID-19. Asimismo, la tasa estimada con fechas de diagnóstico de casos positivos notificados a diario es errada. Del mismo modo, se está calculando incorrectamente la dinámica de infección usando la diferencia de casos positivos en dos días continuos.

El análisis de casos incidentes podría aproximarse y entenderse como velocidad de transmisión, siempre y cuando su definición se fundamente en la ocurrencia del evento y no en la fecha de diagnóstico. Solo cuando la diferencia entre el inicio de síntomas y el diagnóstico es cercana a cero o constante y conocida para toda la población, la fecha diagnóstica podría ser usada como definición de caso incidente. En tal sentido, los casos positivos notificados a diario no deberían ser considerados como casos incidentes debido a que la fecha de diagnóstico reportada es potencialmente afectada por i) la escasez de pruebas diagnósticas; ii) la falta de descentralización de las pruebas en todo nivel; y iii) una posible demora logística para la confirmación de la infección.

Para ilustrar lo expuesto, en la Figura 1A se presenta la curva epidémica de casos incidentes y en $1 \mathrm{~B}$, la incidencia acumulada para China y Japón. Al comparar la distribución acumulada de los casos incidentes, según el inicio de síntomas (azul) y el día de confirmación (amarillo), observamos un retraso promedio de siete días para el periodo de análisis. Si en dichas fechas ajustamos los datos a un modelo exponencial, se proyectarían escenarios con tiempos de duplicación de 0,56 días y 7,20 días para China y Japón, respectivamente. Además, al usar la curva acumulada como una «proyección», solo se presentaría una «aparente» reducción cuando los casos incidentes ya han disminuido en su mayoría. La confirmación de casos de COVID-19 ha demorado, 
A
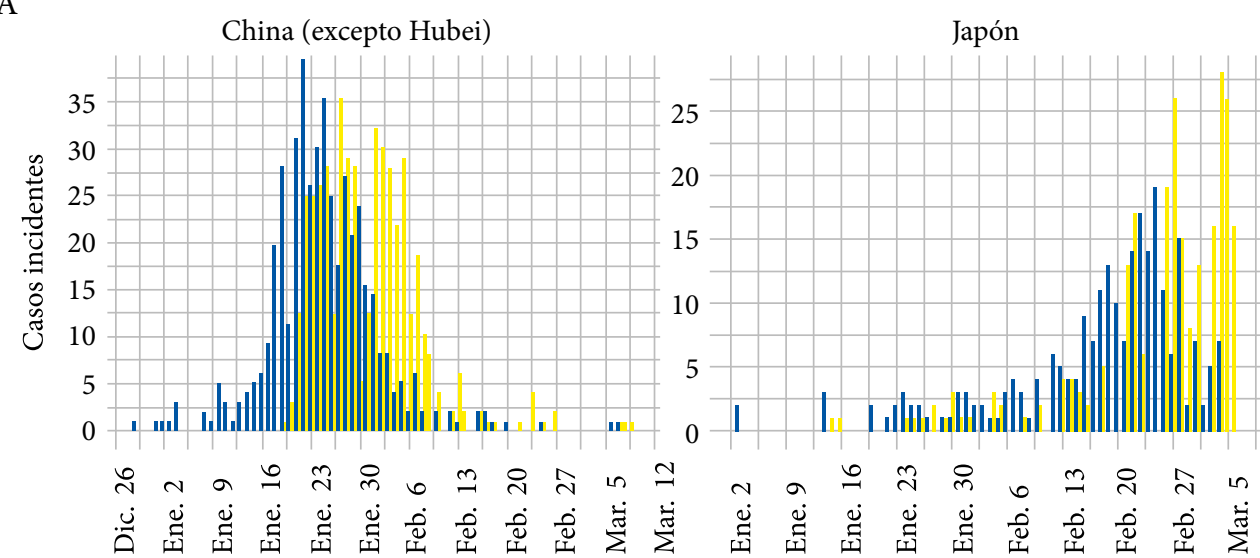

Día calendario

B

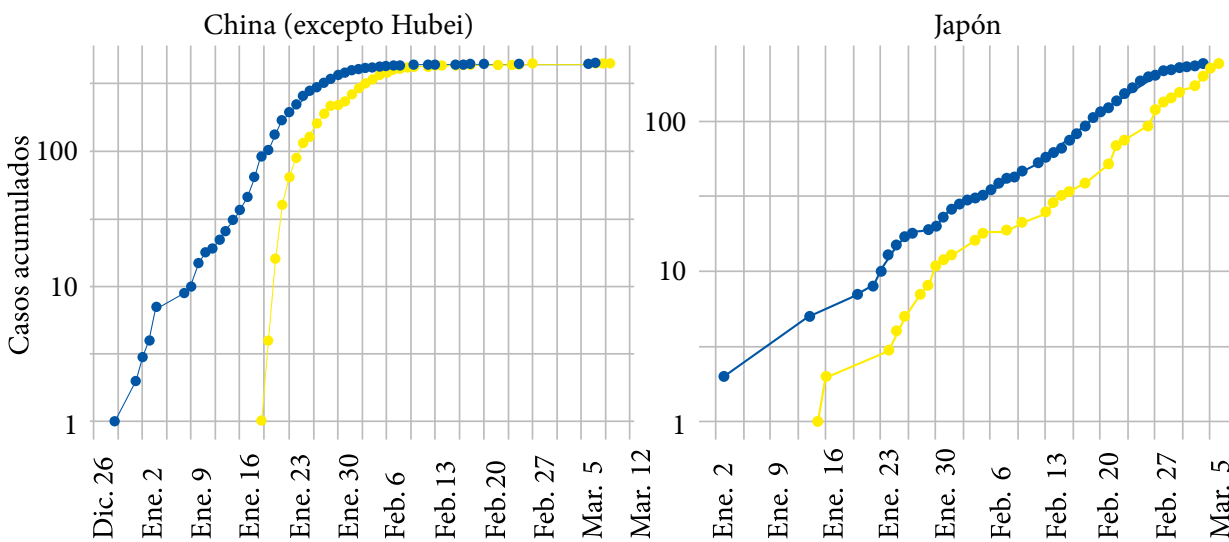

Día calendario

C
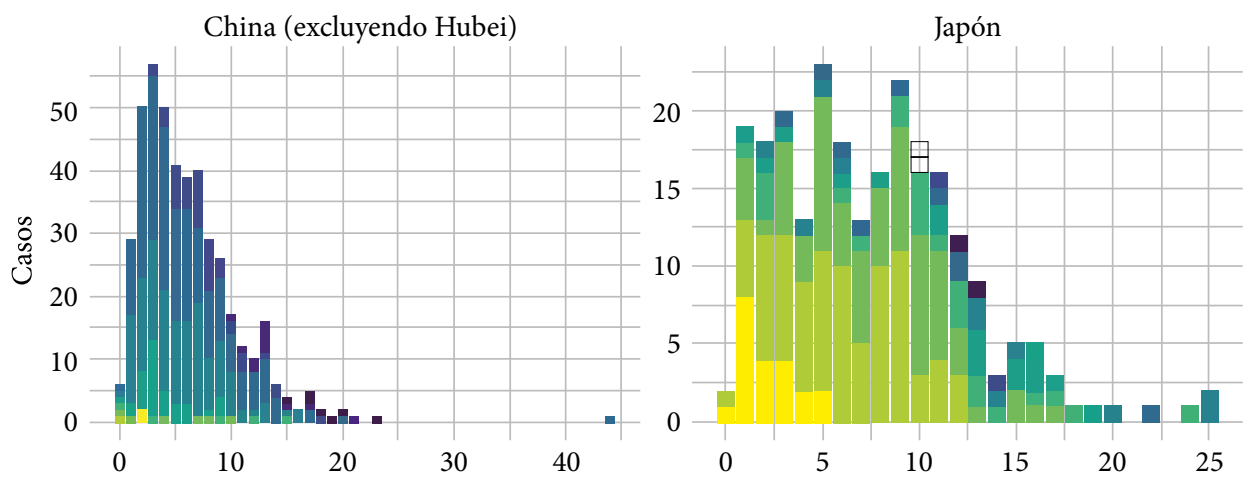

Semana de inicio de síntomas

Tiempo del inicio de síntomas a la confirmación del caso (días)

Figura 1. Distribuciones de casos de COVID-19 según el inicio de síntomas (azul), la confirmación de caso (amarillo) y el tiempo de demora entre ambas a nivel individual en China (excepto la provincia de Hubei) y Japón en el año 2020. (A) En azul: curva de casos incidentes definidos por el inicio de síntomas (curva epidémica); en amarillo: curva de casos confirmados por día, según la proporción de personas diagnosticadas en la población de casos sospechosos. (B) Curva de casos acumulados en escala logarítmica con los mismos datos. (C) Tiempo de demora entre el inicio de síntomas y la confirmación del caso, según las semanas epidemiológicas

con una mediana de 5 (rango intercuartil [RI]: 3-8) días en China y de 7 (RI: 3-11) días en Japón, la cual se ha ido reduciendo con el trascurrir de las semanas hasta una mediana de 2 (RI: 1-3) días en nueve semanas (Figura 1C). En ambos escenarios la diferencia de días entre el inicio de síntomas y la fecha de diagnóstico no ha sido constante y tendió a disminuir conforme la epidemia avanzaba. Por tanto, la fecha de diagnóstico no debe ser usada como definición de caso incidente y tampoco como una aproximación de la tasa de incidencia ni de dinámica de infección. 
En conclusión, felicitamos la iniciativa para analizar datos en tiempo real, pues ello es reflejo del interés creciente sobre el impacto de la COVID-19 en nuestra población. No obstante, recomendamos que los gráficos presentados sean acompañados de una interpretación adecuada, limitaciones y conclusiones respaldadas en el análisis ejecutado y en la naturaleza de los datos utilizados.

Contribuciones de los autores: SL y AV participaron en la concepción y diseño del artículo. Los datos fueron recolectados y analizados por AV, e interpretados por SL, AV, SM y GCE. Todos los autores participaron en la redacción del artículo, revisión crítica y aprobación de la versión final.

Financiamiento: El estudio fue autofinanciado.

Conflictos de interés: Los autores declaran no tener conflicto de intereses

Material suplementario: Disponible en la versión electrónica de la RPMESP

\section{REFERENCIAS BIBLIOGRÁFICAS}

1. World Heatlh Organitzation [Internet]. Rolling updates on coronovirus disease (COVID-19). [citado el 9 de abril de 2020]. Disponible en: https:// www.who.int/emergencies/diseases/novel-coronavirus-2019/events-asthey-happen.

2. Xu B, Kraemer MUG; Open COVID-19 Data Curation Group. Open access epidemiological data from the COVID-19 outbreak. Lancet Infect Dis. 2020;S1473-3099(20)30119-5. doi: 10.1016/S1473-3099(20)30119-5.

3. Andree Valle [Internet]. Lima: Repositorio; 2020 [citado el 9 de abril de 2020] Disponible en: https://github.com/avallecam/covid19_peru_curvas.

4. King AA, Domenech de Cellès M, Magpantay FM, Rohani P. Avoidable errors in the modelling of outbreaks of emerging pathogens, with special reference to Ebola. Proc Biol Sci. 2015;282(1806):20150347. doi: 10.1098/rspb.2015.0347.

5. Karako K, Song P, Chen Y, Tang W. Analysis of COVID-19 infection spread in Japan based on stochastic transition model [published online ahead of print, 2020 Mar 19]. Biosci Trends. 2020;10.5582/bst.2020.01482. doi: 10.5582/bst.2020.01482.

6. Razum O, Becher H, Kapaun A, Junghanss T. SARS, lay epidemiology, and fear. Lancet. 2003;361(9370):1739-1740. doi: 10.1016/S01406736(03)13335-1. 